\title{
A colorimetric electronic tongue for rapid discrimination of antioxidants based on the oxidation etching of nano-triangular silver by metal ions
}

\author{
Siqun Li, ${ }^{\dagger} \mathrm{Kai} \mathrm{Li},{ }^{\dagger}$ Xin Li, Zhengbo Chen* \\ Department of Chemistry, Capital Normal University, Beijing, 100048, China \\ E-mail: czb979216@sina.com \\ $\uparrow$ These authors contribute this work equally
}




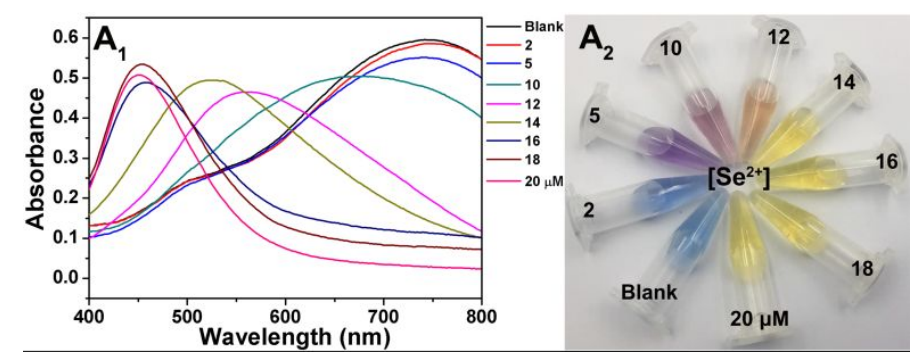

Figure S1. (A) UV-vis spectra, and (B) photographs of TriAgNPs in the presence of different concentrations of $\mathrm{Se}^{2+}$. 


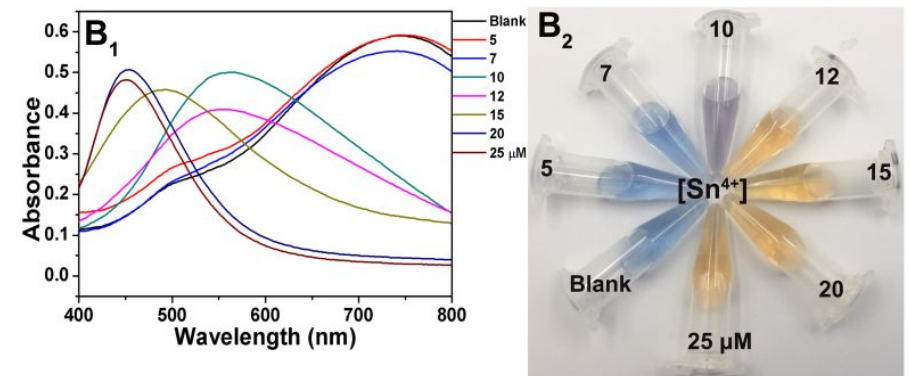

Figure S2. (A) UV-vis spectra, and (B) photographs of TriAgNPs in the presence of different concentrations of $\mathrm{Sn}^{4+}$. 


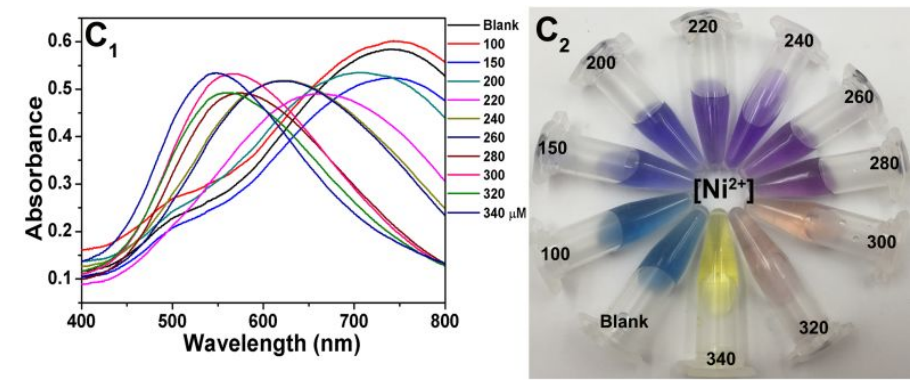

Figure S3. (A) UV-vis spectra, and (B) photographs of TriAgNPs in the presence of different concentrations of $\mathrm{Ni}^{2+}$. 


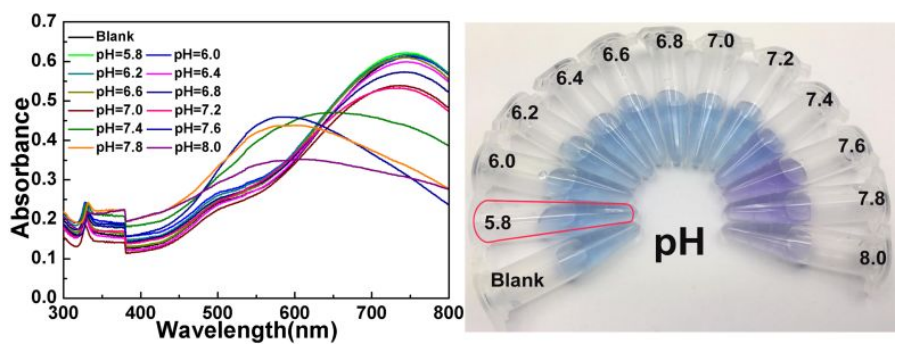

Figure S4. (A) UV-vis spectra, and (B) photographs of TriAgNPs in the presence of different $\mathrm{pH}$ environment. 
Table S1 The training matrix of the colorimetric response patterns against 6 antioxidants by using this sensor assay at the concentration of $200 \mathrm{nM}$.

\begin{tabular}{|c|c|c|c|}
\hline Antioxidants & $\begin{array}{c}\mathrm{Se}^{2+} \\
\lambda_{\mathrm{i}}-\lambda_{0}\end{array}$ & $\begin{array}{c}\mathrm{Sn}^{4+} \\
\lambda_{\mathrm{i}}-\lambda_{0}\end{array}$ & $\begin{array}{l}\mathrm{Ni}^{2+} \\
\lambda_{\mathrm{i}}-\lambda_{0}\end{array}$ \\
\hline EDTA & -16.5 & -10 & -40 \\
\hline EDTA & -23 & -11 & -44 \\
\hline EDTA & -24 & -13 & -44 \\
\hline EDTA & -22 & -13 & -44 \\
\hline EDTA & -26 & -13 & -42 \\
\hline TA & 20 & -11 & 16 \\
\hline TA & 22 & -14 & 14 \\
\hline TA & 20 & -13 & 14 \\
\hline TA & 17 & -13 & 14 \\
\hline TA & 19 & -13 & 16 \\
\hline Gly & 55 & 17 & 34 \\
\hline Gly & 55 & 17 & 32 \\
\hline Gly & 58 & 15 & 32 \\
\hline Gly & 53 & 15 & 32 \\
\hline Gly & 49 & 16 & 34 \\
\hline $\mathrm{AA}$ & 22 & 28 & 182 \\
\hline $\mathrm{AA}$ & 24 & 27 & 176 \\
\hline $\mathrm{AA}$ & 24 & 23 & 174 \\
\hline $\mathrm{AA}$ & 20 & 25 & 176 \\
\hline AA & 23 & 28 & 178 \\
\hline $\mathrm{TP}$ & 37 & 49 & -4 \\
\hline $\mathrm{TP}$ & 38 & 52 & 0 \\
\hline $\mathrm{TP}$ & 37 & 43 & -4 \\
\hline $\mathrm{TP}$ & 35 & 46 & -6 \\
\hline $\mathrm{TP}$ & 35 & 52 & -2 \\
\hline $\mathrm{CA}$ & 28 & 17 & 30 \\
\hline $\mathrm{CA}$ & 32 & 16 & 26 \\
\hline $\mathrm{CA}$ & 29 & 15 & 26 \\
\hline $\mathrm{CA}$ & 28 & 14 & 26 \\
\hline $\mathrm{CA}$ & 26 & 16 & 28 \\
\hline
\end{tabular}


Table S2 The training matrix of the colorimetric response patterns against 6 antioxidants by using this sensor assay at the concentration of $150 \mathrm{nM}$.

\begin{tabular}{|c|c|c|c|}
\hline Antioxidants & $\begin{array}{c}\mathrm{Se}^{2+} \\
\lambda_{\mathrm{i}}-\lambda_{0}\end{array}$ & $\begin{array}{c}\mathrm{Sn}^{4+} \\
\lambda_{\mathrm{i}}-\lambda_{0}\end{array}$ & $\begin{array}{l}\mathrm{Ni}^{2+} \\
\lambda_{\mathrm{i}}-\lambda_{0}\end{array}$ \\
\hline EDTA & -66 & -32 & 62 \\
\hline EDTA & -62 & -32 & 62 \\
\hline EDTA & -60 & -30 & 64 \\
\hline EDTA & -64 & -32 & 68 \\
\hline EDTA & -58 & -32 & 66 \\
\hline TA & -22 & 24 & 72 \\
\hline TA & -22 & 24 & 74 \\
\hline TA & -28 & 24 & 76 \\
\hline TA & -24 & 26 & 78 \\
\hline TA & -24 & 28 & 84 \\
\hline Gly & -6 & 10 & 0 \\
\hline Gly & -4 & 10 & 2 \\
\hline Gly & -8 & 10 & -2 \\
\hline Gly & -4 & 8 & 0 \\
\hline Gly & -6 & 12 & 2 \\
\hline $\mathrm{AA}$ & -24 & 0 & 84 \\
\hline $\mathrm{AA}$ & -32 & 0 & 80 \\
\hline AA & -34 & 0 & 84 \\
\hline AA & -28 & 0 & 88 \\
\hline AA & -26 & 0 & 86 \\
\hline $\mathrm{TP}$ & -16 & 12 & 54 \\
\hline $\mathrm{TP}$ & -16 & 12 & 54 \\
\hline $\mathrm{TP}$ & -18 & 12 & 52 \\
\hline $\mathrm{TP}$ & -16 & 12 & 60 \\
\hline $\mathrm{TP}$ & -20 & 12 & 62 \\
\hline CA & -14 & 18 & 62 \\
\hline $\mathrm{CA}$ & -14 & 18 & 66 \\
\hline $\mathrm{CA}$ & -16 & 16 & 70 \\
\hline $\mathrm{CA}$ & -14 & 16 & 68 \\
\hline $\mathrm{CA}$ & -14 & 18 & 70 \\
\hline
\end{tabular}


Table S3 The training matrix of the colorimetric response patterns against 6 antioxidants by using this sensor assay at the concentration of $100 \mathrm{nM}$.

\begin{tabular}{|c|c|c|c|}
\hline Antioxidants & $\begin{array}{c}\mathrm{Se}^{2+} \\
\lambda_{\mathrm{i}}-\lambda_{0}\end{array}$ & $\begin{array}{c}\mathrm{Sn}^{4+} \\
\lambda_{\mathrm{i}}-\lambda_{0}\end{array}$ & $\begin{array}{c}\mathrm{Ni}^{2+} \\
\lambda_{\mathrm{i}}-\lambda_{0}\end{array}$ \\
\hline EDTA & 70 & -42 & 6 \\
\hline EDTA & 75 & -36 & 2 \\
\hline EDTA & 70 & -38 & 2 \\
\hline EDTA & 68 & -38 & 2 \\
\hline EDTA & 73 & -42 & 4 \\
\hline TA & -34 & 4 & 4 \\
\hline TA & -34 & 6 & 2 \\
\hline TA & -34 & 10 & 2 \\
\hline TA & -38 & 4 & 4 \\
\hline TA & -34 & 6 & 4 \\
\hline Gly & -50 & -6 & -6 \\
\hline Gly & -44 & -2 & -8 \\
\hline Gly & -48 & -4 & -4 \\
\hline Gly & -50 & -6 & -10 \\
\hline Gly & -42 & -2 & -6 \\
\hline $\mathrm{AA}$ & -26 & -22 & -6 \\
\hline $\mathrm{AA}$ & -34 & -14 & -8 \\
\hline AA & -34 & -18 & -6 \\
\hline AA & -38 & -20 & -6 \\
\hline AA & -34 & -18 & -8 \\
\hline $\mathrm{TP}$ & -20 & -16 & -78 \\
\hline $\mathrm{TP}$ & -20 & -14 & -80 \\
\hline $\mathrm{TP}$ & -22 & -16 & -80 \\
\hline $\mathrm{TP}$ & -24 & -16 & -80 \\
\hline $\mathrm{TP}$ & -24 & -18 & -78 \\
\hline $\mathrm{CA}$ & -24 & 0 & -6 \\
\hline $\mathrm{CA}$ & -22 & -4 & -10 \\
\hline $\mathrm{CA}$ & -24 & -6 & -8 \\
\hline $\mathrm{CA}$ & -26 & -6 & -6 \\
\hline $\mathrm{CA}$ & -24 & -6 & -4 \\
\hline
\end{tabular}


Table S4 The training matrix of the colorimetric response patterns against 6 antioxidants by using this sensor assay at the concentration of $50 \mathrm{nM}$.

\begin{tabular}{|c|c|c|c|}
\hline Antioxidants & $\begin{array}{c}\mathrm{Se}^{2+} \\
\lambda_{\mathrm{i}}-\lambda_{0}\end{array}$ & $\begin{array}{c}\mathrm{Sn}^{4+} \\
\lambda_{\mathrm{i}}-\lambda_{0}\end{array}$ & $\begin{array}{l}\mathrm{Ni}^{2+} \\
\lambda_{\mathrm{i}}-\lambda_{0}\end{array}$ \\
\hline EDTA & -84 & -52 & -74 \\
\hline EDTA & -84 & -50 & -75 \\
\hline EDTA & -84 & -46 & -73 \\
\hline EDTA & -80 & -46 & -74 \\
\hline EDTA & -84 & -46 & -74 \\
\hline TA & -72 & -50 & -340 \\
\hline TA & -72 & -48 & -339 \\
\hline TA & -72 & -46 & -341 \\
\hline TA & -70 & -46 & -341 \\
\hline TA & -70 & -44 & -340 \\
\hline Gly & -32 & -18 & -4 \\
\hline Gly & -32 & -14 & -3 \\
\hline Gly & -34 & -16 & -5 \\
\hline Gly & -28 & -14 & -3 \\
\hline Gly & -30 & -14 & -4 \\
\hline $\mathrm{AA}$ & 30 & -12 & -176 \\
\hline $\mathrm{AA}$ & 30 & -14 & -181 \\
\hline $\mathrm{AA}$ & 26 & -14 & -183 \\
\hline $\mathrm{AA}$ & 30 & -12 & -180 \\
\hline AA & 30 & -12 & -180 \\
\hline $\mathrm{TP}$ & 96 & -88 & 16 \\
\hline $\mathrm{TP}$ & 94 & -86 & 12 \\
\hline $\mathrm{TP}$ & 96 & -86 & 12 \\
\hline $\mathrm{TP}$ & 100 & -84 & 16 \\
\hline $\mathrm{TP}$ & 94 & -84 & 16 \\
\hline CA & -22 & -34 & 48 \\
\hline $\mathrm{CA}$ & -22 & -32 & 47 \\
\hline $\mathrm{CA}$ & -20 & -32 & 48 \\
\hline $\mathrm{CA}$ & -18 & -32 & 47 \\
\hline $\mathrm{CA}$ & -24 & -32 & 48 \\
\hline
\end{tabular}


Table S5 The training matrix of the colorimetric response patterns against 6 antioxidants by using this sensor assay at the concentration of $30 \mathrm{nM}$.

\begin{tabular}{|c|c|c|c|}
\hline Antioxidants & $\begin{array}{c}\mathrm{Se}^{2+} \\
\lambda_{\mathrm{i}}-\lambda_{0}\end{array}$ & $\begin{array}{c}\mathrm{Sn}^{4+} \\
\lambda_{\mathrm{i}}-\lambda_{0}\end{array}$ & $\begin{array}{l}\mathrm{Ni}^{2+} \\
\lambda_{\mathrm{i}}-\lambda_{0}\end{array}$ \\
\hline EDTA & -24 & -36 & 4 \\
\hline EDTA & -22 & -36 & 2 \\
\hline EDTA & -24 & -38 & 8 \\
\hline EDTA & -20 & -38 & 6 \\
\hline EDTA & -18 & -36 & 6 \\
\hline TA & -2 & -32 & 0 \\
\hline TA & -2 & -32 & 0 \\
\hline TA & -2 & -34 & 4 \\
\hline TA & -2 & -32 & 2 \\
\hline TA & 0 & -36 & 2 \\
\hline Gly & -2 & -44 & -116 \\
\hline Gly & -2 & -44 & -118 \\
\hline Gly & -2 & -48 & -114 \\
\hline Gly & -2 & -46 & -116 \\
\hline Gly & 0 & -44 & -120 \\
\hline $\mathrm{AA}$ & -12 & -30 & -20 \\
\hline $\mathrm{AA}$ & -10 & -28 & -20 \\
\hline AA & -8 & -32 & -20 \\
\hline AA & -10 & -32 & -22 \\
\hline AA & -10 & -32 & -22 \\
\hline $\mathrm{TP}$ & -62 & -164 & -148 \\
\hline $\mathrm{TP}$ & -60 & -164 & -148 \\
\hline $\mathrm{TP}$ & -60 & -165 & -148 \\
\hline $\mathrm{TP}$ & -62 & -163 & -150 \\
\hline $\mathrm{TP}$ & -62 & -165 & -148 \\
\hline CA & 18 & -10 & -144 \\
\hline $\mathrm{CA}$ & 16 & -10 & -144 \\
\hline $\mathrm{CA}$ & 18 & -12 & -142 \\
\hline $\mathrm{CA}$ & 16 & -10 & -143 \\
\hline $\mathrm{CA}$ & 16 & -10 & -143 \\
\hline
\end{tabular}


Table S6 The training matrix of the colorimetric response patterns against 6 antioxidants by using this sensor assay at the concentration of $10 \mathrm{nM}$.

\begin{tabular}{|c|c|c|c|}
\hline Antioxidants & $\begin{array}{c}\mathrm{Se}^{2+} \\
\lambda_{\mathrm{i}}-\lambda_{0}\end{array}$ & $\begin{array}{c}\mathrm{Sn}^{4+} \\
\lambda_{\mathrm{i}}-\lambda_{0} \\
\end{array}$ & $\begin{array}{c}\mathrm{Ni}^{2+} \\
\lambda_{\mathrm{i}}-\lambda_{0}\end{array}$ \\
\hline EDTA & -4 & -4 & -8 \\
\hline EDTA & -6 & -2 & -6 \\
\hline EDTA & -6 & -2 & -4 \\
\hline EDTA & -4 & -2 & -4 \\
\hline EDTA & -4 & -2 & -4 \\
\hline TA & -26 & 10 & -14 \\
\hline TA & -30 & 8 & -16 \\
\hline TA & -28 & 8 & -14 \\
\hline TA & -28 & 8 & -12 \\
\hline TA & -30 & 8 & -10 \\
\hline Gly & -26 & 12 & -10 \\
\hline Gly & -26 & 14 & -8 \\
\hline Gly & -26 & 14 & -8 \\
\hline Gly & -24 & 12 & -5 \\
\hline Gly & -24 & 14 & -4 \\
\hline AA & -24 & 46 & 0 \\
\hline AA & -28 & 46 & 0 \\
\hline AA & -26 & 46 & 4 \\
\hline AA & -24 & 48 & 4 \\
\hline AA & -26 & 48 & 6 \\
\hline $\mathrm{TP}$ & -42 & 0 & 84 \\
\hline $\mathrm{TP}$ & -44 & 0 & 84 \\
\hline $\mathrm{TP}$ & -44 & 0 & 88 \\
\hline $\mathrm{TP}$ & -44 & 2 & 86 \\
\hline $\mathrm{TP}$ & -44 & 0 & 88 \\
\hline CA & -28 & -2 & 0 \\
\hline $\mathrm{CA}$ & -32 & -4 & 0 \\
\hline $\mathrm{CA}$ & -32 & -2 & 2 \\
\hline $\mathrm{CA}$ & -30 & -4 & 4 \\
\hline $\mathrm{CA}$ & -32 & -4 & 4 \\
\hline
\end{tabular}


Table S7 The training matrix of the colorimetric response patterns against 6 antioxidants by using this sensor assay at the concentration of $1 \mathrm{nM}$.

\begin{tabular}{|c|c|c|c|}
\hline Antioxidants & $\begin{array}{c}\mathrm{Se}^{2+} \\
\lambda_{\mathrm{i}}-\lambda_{0}\end{array}$ & $\begin{array}{c}\mathrm{Sn}^{4+} \\
\lambda_{\mathrm{i}}-\lambda_{0}\end{array}$ & $\begin{array}{l}\mathrm{Ni}^{2+} \\
\lambda_{\mathrm{i}}-\lambda_{0}\end{array}$ \\
\hline EDTA & -42 & 6 & 30 \\
\hline EDTA & -42 & 10 & 34 \\
\hline EDTA & -38 & 10 & 29 \\
\hline EDTA & -42 & 12 & 30 \\
\hline EDTA & -40 & 10 & 31 \\
\hline TA & -42 & -4 & 28 \\
\hline TA & -42 & -4 & 32 \\
\hline TA & -40 & -4 & 30 \\
\hline TA & -42 & -4 & 31 \\
\hline TA & -40 & -2 & 32 \\
\hline Gly & -12 & -16 & -66 \\
\hline Gly & -12 & -14 & -60 \\
\hline Gly & -10 & -16 & -64 \\
\hline Gly & -12 & -14 & -64 \\
\hline Gly & -8 & -12 & -64 \\
\hline $\mathrm{AA}$ & -18 & -2 & -77 \\
\hline $\mathrm{AA}$ & -16 & -2 & -74 \\
\hline AA & -16 & -2 & -76 \\
\hline AA & -18 & -2 & -74 \\
\hline AA & -16 & -2 & -76 \\
\hline $\mathrm{TP}$ & 16 & -24 & 30 \\
\hline $\mathrm{TP}$ & 18 & -22 & 30 \\
\hline $\mathrm{TP}$ & 18 & -24 & 30 \\
\hline $\mathrm{TP}$ & 16 & -22 & 32 \\
\hline $\mathrm{TP}$ & 16 & -23 & 31 \\
\hline CA & -20 & 2 & 18 \\
\hline $\mathrm{CA}$ & -20 & 6 & 22 \\
\hline $\mathrm{CA}$ & -18 & 4 & 18 \\
\hline $\mathrm{CA}$ & -22 & 4 & 22 \\
\hline $\mathrm{CA}$ & -20 & 6 & 20 \\
\hline
\end{tabular}


Table S8. Comparison of different sensor arrays for discrimination of antioxidants.

\begin{tabular}{|l|l|l|l|}
\hline method & recognition number & LOD & ref. \\
\hline colorimetric & 6 & $1 \mathrm{nM}$ & this work \\
colorimetric & 5 & $1 \mu \mathrm{M}$ & {$[1]$} \\
colorimetric & 5 & $20 \mu \mathrm{M}$ & {$[2]$} \\
colorimetric & 5 & $60 \mathrm{nM}$ & {$[3]$} \\
fluorescent & 8 & $0.7 \mu \mathrm{M}$ & {$[4]$} \\
fluorescent & 5 & $50 \mathrm{nM}$ & {$[5]$} \\
\hline
\end{tabular}

\section{References}

[1] Huang, W.; Xie, Z. Y.; Deng, Y. Q.; He, Y. 3,3' ,5,5' -tetramethylbenzidine-Based Quadruple-Channel Visual Colorimetric Sensor Array for Highly Sensitive Discrimination of Serum Antioxidants. Sens. Actuat. B 2018, 254, 1057-1060.

[2] Huang, W.; Deng, Y. Q.; He, Y. Visual Colorimetric Sensor Array for Discrimination of Antioxidants in Serum using $\mathrm{MnO}_{2}$ Nanosheets Triggered Multicolor Chromogenic System. Biosens. Bioelectron. 2017, 91, 89-94.

[3] Li, X.; Kong, C. Y.; Chen, Z. B. Colorimetric Sensor Arrays for Antioxidant Discrimination Based on the Inhibition of the Oxidation Reaction between 3,3',5,5'-Tetramethylbenzidine and Hydrogen Peroxides. ACS Appl. Mater. Interfaces 2019,11, 9504-9509.

[4] Liu, H. L.; Fang, G. Z.; Deng, Q. L.; Wang, S. A Triple-Dimensional Sensing Chip for Discrimination of Eight Antioxidants Based on Quantum Dots and Graphene. Biosens. Bioelectron. 2015, 74, 313-317.

[5] Xi, H. Y.; Li, X.; Liu, Q. Y.; Chen, Z. B. Fluorescent Sensor Array for Discrimination of Biothiols Based on Poly(Thymine/Cytosine)-Templated Copper Nanoparticles. Anal. Chim. Acta 2019, 1051, 147-152. 
Table S9 The training matrix of the colorimetric response patterns against the different antioxidants at different concentrations by using this sensor assay.

\begin{tabular}{|c|c|c|c|}
\hline Antioxidants & $\begin{array}{c}\mathrm{Se}^{2+} \\
\lambda_{\mathrm{i}}-\lambda_{0}\end{array}$ & $\begin{array}{c}\mathrm{Sn}^{4+} \\
\lambda_{\mathrm{i}}-\lambda_{0}\end{array}$ & $\begin{array}{c}\mathrm{Ni}^{2+} \\
\lambda_{\mathrm{i}}-\lambda_{0}\end{array}$ \\
\hline $\operatorname{EDTA}(30 \mathrm{nM})$ & -58 & -26 & 4 \\
\hline $\operatorname{EDTA}(30 \mathrm{nM})$ & -58 & -28 & 2 \\
\hline $\operatorname{EDTA}(30 \mathrm{nM})$ & -56 & -26 & 8 \\
\hline $\operatorname{EDTA}(30 \mathrm{nM})$ & -58 & -24 & 6 \\
\hline $\operatorname{EDTA}(30 \mathrm{nM})$ & -56 & -26 & 6 \\
\hline $\operatorname{EDTA}(150$ nM) & -46 & -2 & -40 \\
\hline EDTA(150 nM) & -48 & -4 & -44 \\
\hline EDTA(150 nM) & -44 & -4 & -44 \\
\hline EDTA(150 nM) & -48 & -4 & -44 \\
\hline EDTA(150 nM) & -46 & -2 & -42 \\
\hline $\operatorname{EDTA}(200$ nM) & -40 & -2 & -74 \\
\hline $\operatorname{EDTA}(200$ nM) & -42 & -4 & -75 \\
\hline EDTA(200 nM) & -38 & -2 & -73 \\
\hline EDTA(200 nM) & -42 & 0 & -74 \\
\hline $\operatorname{EDTA}(200 \mathrm{nM})$ & -42 & 0 & -74 \\
\hline $\mathrm{TA}(10 \mathrm{nM})$ & -26 & 10 & -14 \\
\hline $\mathrm{TA}(10 \mathrm{nM})$ & -30 & 8 & -16 \\
\hline $\mathrm{TA}(10 \mathrm{nM})$ & -28 & 8 & -14 \\
\hline $\mathrm{TA}(10 \mathrm{nM})$ & -28 & 8 & -12 \\
\hline $\mathrm{TA}(10 \mathrm{nM})$ & -30 & 8 & -10 \\
\hline $\mathrm{TA}(100 \mathrm{nM})$ & -42 & -4 & 28 \\
\hline $\mathrm{TA}(100 \mathrm{nM})$ & -42 & -4 & 32 \\
\hline $\mathrm{TA}(100 \mathrm{nM})$ & -40 & -4 & 30 \\
\hline $\mathrm{TA}(100 \mathrm{nM})$ & -42 & -4 & 31 \\
\hline $\mathrm{TA}(100 \mathrm{nM})$ & -40 & -2 & 32 \\
\hline $\mathrm{TA}(150 \mathrm{nM})$ & 20 & -11 & 16 \\
\hline $\mathrm{TA}(150 \mathrm{nM})$ & 22 & -14 & 14 \\
\hline $\mathrm{TA}(150 \mathrm{nM})$ & 20 & -13 & 14 \\
\hline $\mathrm{TA}(150 \mathrm{nM})$ & 17 & -13 & 14 \\
\hline $\mathrm{TA}(150 \mathrm{nM})$ & 19 & -13 & 16 \\
\hline $\mathrm{TA}(200 \mathrm{nM})$ & -22 & 24 & 72 \\
\hline $\mathrm{TA}(200 \mathrm{nM})$ & -22 & 24 & 74 \\
\hline $\mathrm{TA}(200 \mathrm{nM})$ & -28 & 24 & 76 \\
\hline $\mathrm{TA}(200 \mathrm{nM})$ & -24 & 26 & 78 \\
\hline $\mathrm{TA}(200 \mathrm{nM})$ & -24 & 28 & 84 \\
\hline Gly(30 nM) & -2 & -44 & -116 \\
\hline Gly(30 nM) & -2 & -44 & -118 \\
\hline Gly(30 nM) & -2 & -48 & -114 \\
\hline Gly(30 nM) & -2 & -46 & -116 \\
\hline Gly(30 nM) & 0 & -44 & -120 \\
\hline
\end{tabular}




\begin{tabular}{|c|c|c|c|}
\hline Gly(50 nM) & -32 & -18 & -4 \\
\hline Gly(50 nM) & -32 & -14 & -3 \\
\hline Gly(50 nM) & -34 & -16 & -5 \\
\hline Gly(50 nM) & -28 & -14 & -3 \\
\hline Gly(50 nM) & -30 & -14 & -4 \\
\hline Gly(100 nM) & -50 & -6 & -6 \\
\hline Gly(100 nM) & -44 & -2 & -8 \\
\hline Gly(100 nM) & -48 & -4 & -4 \\
\hline Gly(100 nM) & -50 & -6 & -10 \\
\hline Gly(100 nM) & -42 & -2 & -6 \\
\hline $\mathrm{AA}(50 \mathrm{nM})$ & 30 & -12 & -176 \\
\hline $\mathrm{AA}(50 \mathrm{nM})$ & 30 & -14 & -181 \\
\hline $\mathrm{AA}(50 \mathrm{nM})$ & 26 & -14 & -183 \\
\hline $\mathrm{AA}(50 \mathrm{nM})$ & 30 & -12 & -180 \\
\hline $\mathrm{AA}(50 \mathrm{nM})$ & 30 & -12 & -180 \\
\hline $\mathrm{AA}(150 \mathrm{nM})$ & -24 & 0 & 84 \\
\hline $\mathrm{AA}(150 \mathrm{nM})$ & -32 & 0 & 80 \\
\hline $\mathrm{AA}(150 \mathrm{nM})$ & -34 & 0 & 84 \\
\hline $\mathrm{AA}(150 \mathrm{nM})$ & -28 & 0 & 88 \\
\hline $\mathrm{AA}(150 \mathrm{nM})$ & -26 & 0 & 86 \\
\hline $\mathrm{AA}(200 \mathrm{nM})$ & 22 & 28 & 182 \\
\hline $\mathrm{AA}(200 \mathrm{nM})$ & 24 & 27 & 176 \\
\hline $\mathrm{AA}(200 \mathrm{nM})$ & 24 & 23 & 174 \\
\hline $\mathrm{AA}(200 \mathrm{nM})$ & 20 & 25 & 176 \\
\hline $\mathrm{AA}(200 \mathrm{nM})$ & 23 & 28 & 178 \\
\hline $\mathrm{TP}(50 \mathrm{nM})$ & 96 & -88 & 16 \\
\hline $\mathrm{TP}(50 \mathrm{nM})$ & 94 & -86 & 12 \\
\hline $\mathrm{TP}(50 \mathrm{nM})$ & 96 & -86 & 12 \\
\hline $\mathrm{TP}(50 \mathrm{nM})$ & 100 & -84 & 16 \\
\hline $\mathrm{TP}(50 \mathrm{nM})$ & 94 & -84 & 16 \\
\hline $\mathrm{TP}(100 \mathrm{nM})$ & 16 & -24 & 30 \\
\hline $\mathrm{TP}(100 \mathrm{nM})$ & 18 & -22 & 30 \\
\hline $\mathrm{TP}(100 \mathrm{nM})$ & 18 & -24 & 30 \\
\hline $\mathrm{TP}(100 \mathrm{nM})$ & 16 & -22 & 32 \\
\hline $\mathrm{TP}(100 \mathrm{nM})$ & 16 & -23 & 31 \\
\hline $\mathrm{TP}(150 \mathrm{nM})$ & -16 & 12 & 54 \\
\hline $\mathrm{TP}(150 \mathrm{nM})$ & -16 & 12 & 54 \\
\hline $\mathrm{TP}(150 \mathrm{nM})$ & -18 & 12 & 52 \\
\hline $\mathrm{TP}(150 \mathrm{nM})$ & -16 & 12 & 60 \\
\hline $\mathrm{TP}(150 \mathrm{nM})$ & -20 & 12 & 62 \\
\hline $\mathrm{TP}(200 \mathrm{nM})$ & 37 & 49 & -4 \\
\hline $\mathrm{TP}(200 \mathrm{nM})$ & 38 & 52 & 0 \\
\hline $\mathrm{TP}(200 \mathrm{nM})$ & 37 & 43 & -4 \\
\hline $\mathrm{TP}(200 \mathrm{nM})$ & 35 & 46 & -6 \\
\hline
\end{tabular}




\begin{tabular}{llll}
\hline $\mathrm{TP}(200 \mathrm{nM})$ & 35 & 52 & -2 \\
$\mathrm{CA}(10 \mathrm{nM})$ & -39 & -6 & -156 \\
$\mathrm{CA}(10 \mathrm{nM})$ & -35 & -7 & -159 \\
$\mathrm{CA}(10 \mathrm{nM})$ & -38 & -8 & -159 \\
$\mathrm{CA}(10 \mathrm{nM})$ & -39 & -9 & -158 \\
$\mathrm{CA}(10 \mathrm{nM})$ & -39 & -9 & -158 \\
$\mathrm{CA}(30 \mathrm{nM})$ & -24 & -38 & -160 \\
$\mathrm{CA}(30 \mathrm{nM})$ & -22 & -38 & -159 \\
$\mathrm{CA}(30 \mathrm{nM})$ & -24 & -42 & -157 \\
$\mathrm{CA}(30 \mathrm{nM})$ & -26 & -42 & -162 \\
$\mathrm{CA}(30 \mathrm{nM})$ & -24 & -40 & -162 \\
$\mathrm{CA}(100 \mathrm{nM})$ & -58 & -30 & -38 \\
$\mathrm{CA}(100 \mathrm{nM})$ & -56 & -28 & -39 \\
$\mathrm{CA}(100 \mathrm{nM})$ & -58 & -30 & -39 \\
$\mathrm{CA}(100 \mathrm{nM})$ & -62 & -32 & -36 \\
$\mathrm{CA}(100 \mathrm{nM})$ & -60 & -30 & -38 \\
$\mathrm{CA}(150 \mathrm{nM})$ & -46 & -48 & -22 \\
$\mathrm{CA}(150 \mathrm{nM})$ & -44 & -48 & -23 \\
$\mathrm{CA}(150 \mathrm{nM})$ & -44 & -50 & -22 \\
$\mathrm{CA}(150 \mathrm{nM})$ & -48 & -50 & -20 \\
$\mathrm{CA}(150 \mathrm{nM})$ & -48 & -50 & -22 \\
$\mathrm{CA}(200 \mathrm{nM})$ & -24 & -44 & 38 \\
$\mathrm{CA}(200 \mathrm{nM})$ & -22 & -50 & 35 \\
$\mathrm{CA}(200 \mathrm{nM})$ & -24 & -52 & 35 \\
$\mathrm{CA}(200 \mathrm{nM})$ & -26 & -52 & 38 \\
$\mathrm{CA}(200 \mathrm{nM})$ & -24 & & 38 \\
\hline & & -50 & \\
\hline
\end{tabular}


Table S10 The training matrix of the colorimetric response patterns against the mixture of antioxidants at $100 \mathrm{nM}$ by using this sensor assay.

\begin{tabular}{|c|c|c|c|}
\hline Antioxidant mixture & $\begin{array}{c}\mathrm{Se}^{2+} \\
\lambda_{\mathrm{i}}-\lambda_{0}\end{array}$ & $\begin{array}{c}\mathrm{Sn}^{4+} \\
\lambda_{\mathrm{i}}-\lambda_{0}\end{array}$ & $\begin{array}{c}\mathrm{Ni}^{2+} \\
\lambda_{\mathrm{i}}-\lambda_{0}\end{array}$ \\
\hline Gly:AA=2:8 & 48 & 16 & 12 \\
\hline Gly:AA=2:8 & 46 & 16 & 12 \\
\hline Gly:AA=2:8 & 46 & 16 & 10 \\
\hline Gly:AA=2:8 & 48 & 16 & 14 \\
\hline Gly:AA=2:8 & 45 & 13 & 13 \\
\hline EDTA:TA $=5: 5$ & -2 & 22 & 36 \\
\hline EDTA:TA $=5: 5$ & -2 & 22 & 34 \\
\hline EDTA:TA $=5: 5$ & -2 & 23 & 34 \\
\hline EDTA:TA $=5: 5$ & -2 & 22 & 32 \\
\hline EDTA:TA=5:5 & -3 & 23 & 38 \\
\hline EDTA:CA=3:7 & 2 & 4 & 44 \\
\hline EDTA:CA=3:7 & 4 & 4 & 42 \\
\hline EDTA:CA=3:7 & 4 & 4 & 40 \\
\hline EDTA:CA=3:7 & 5 & 3 & 42 \\
\hline EDTA:CA=3:7 & 5 & 3 & 44 \\
\hline Gly:AA:TP=2:3:5 & 2 & 2 & 48 \\
\hline Gly:AA:TP=2:3:5 & 2 & 2 & 46 \\
\hline Gly:AA:TP=2:3:5 & 4 & 2 & 48 \\
\hline Gly:AA:TP=2:3:5 & 4 & 2 & 48 \\
\hline Gly:AA:TP=2:3:5 & 1 & 1 & 50 \\
\hline TA:AA:CA=4:2:4 & 14 & 12 & 26 \\
\hline TA:AA:CA=4:2:4 & 14 & 12 & 24 \\
\hline TA:AA:CA=4:2:4 & 14 & 12 & 24 \\
\hline TA:AA:CA=4:2:4 & 14 & 12 & 22 \\
\hline TA:AA:CA=4:2:4 & 11 & 11 & 26 \\
\hline AA:TP:CA=3:6:1 & 14 & -2 & 40 \\
\hline $\mathrm{AA}: \mathrm{TP}: \mathrm{CA}=3: 6: 1$ & 14 & -2 & 38 \\
\hline AA:TP:CA=3:6:1 & 14 & -2 & 38 \\
\hline $\mathrm{AA}: \mathrm{TP}: \mathrm{CA}=3: 6: 1$ & 14 & -2 & 38 \\
\hline AA:TP:CA=3:6:1 & 11 & -1 & 40 \\
\hline \multicolumn{4}{|l|}{ EDTA:Gly:TP:CA= } \\
\hline $2: 2: 3: 3$ & 6 & 2 & 26 \\
\hline \multicolumn{4}{|l|}{ EDTA:Gly:TP:CA= } \\
\hline $2: 2: 3: 3$ & 8 & 2 & 22 \\
\hline \multicolumn{4}{|l|}{ EDTA:Gly:TP:CA= } \\
\hline $2: 2: 3: 3$ & 6 & 2 & 22 \\
\hline \multicolumn{4}{|l|}{ EDTA:Gly:TP:CA= } \\
\hline $2: 2: 3: 3$ & 8 & 2 & 22 \\
\hline \multicolumn{4}{|l|}{ EDTA:Gly:TP:CA= } \\
\hline $2: 2: 3: 3$ & 5 & 1 & 18 \\
\hline
\end{tabular}




\begin{tabular}{|c|c|c|c|}
\hline \multicolumn{4}{|c|}{ EDTA:TA:AA:TP $=$} \\
\hline $3: 2: 2: 3$ & 20 & 12 & 28 \\
\hline \multicolumn{4}{|c|}{ EDTA:TA:AA:TP= } \\
\hline $3: 2: 2: 3$ & 20 & 12 & 28 \\
\hline \multicolumn{4}{|c|}{ EDTA:TA:AA:TP= } \\
\hline $3: 2: 2: 3$ & 21 & 12 & 28 \\
\hline \multicolumn{4}{|c|}{ EDTA:TA:AA:TP= } \\
\hline $3: 2: 2: 3$ & 20 & 12 & 28 \\
\hline \multicolumn{4}{|c|}{ EDTA:TA:AA:TP= } \\
\hline $3: 2: 2: 3$ & 21 & 11 & 32 \\
\hline \multicolumn{4}{|c|}{ EDTA:TA:AA:TP:C } \\
\hline $\mathrm{A}=2: 1: 1: 3: 3$ & 14 & 18 & 42 \\
\hline \multicolumn{4}{|c|}{ EDTA:TA:AA:TP:C } \\
\hline$A=2: 1: 1: 3: 3$ & 14 & 18 & 42 \\
\hline \multicolumn{4}{|c|}{ EDTA:TA:AA:TP:C } \\
\hline$A=2: 1: 1: 3: 3$ & 14 & 18 & 42 \\
\hline \multicolumn{4}{|c|}{ EDTA:TA:AA:TP:C } \\
\hline$A=2: 1: 1: 3: 3$ & 14 & 18 & 38 \\
\hline \multicolumn{4}{|c|}{ EDTA:TA:AA:TP:C } \\
\hline$A=2: 1: 1: 3: 3$ & 13 & 17 & 41 \\
\hline
\end{tabular}


Table S11 The training matrix of the colorimetric response patterns against the 6 antioxidants at $30 \mathrm{nM}$ in serum samples by using this sensor assay.

\begin{tabular}{|c|c|c|c|}
\hline Antioxidants & $\begin{array}{c}\mathrm{Se}^{2+} \\
\lambda_{\mathrm{i}}-\lambda_{0}\end{array}$ & $\begin{array}{c}\mathrm{Sn}^{4+} \\
\lambda_{\mathrm{i}}-\lambda_{0}\end{array}$ & $\begin{array}{c}\mathrm{Ni}^{2+} \\
\lambda_{\mathrm{i}}-\lambda_{0}\end{array}$ \\
\hline Serum & 20 & 34 & 36 \\
\hline Serum & 24 & 30 & 35 \\
\hline Serum & 18 & 33 & 37 \\
\hline Serum & 18 & 30 & 35 \\
\hline Serum & 18 & 32 & 36 \\
\hline EDTA & 42 & 36 & 54 \\
\hline EDTA & 42 & 36 & 54 \\
\hline EDTA & 42 & 35 & 53 \\
\hline EDTA & 42 & 36 & 55 \\
\hline EDTA & 42 & 37 & 53 \\
\hline TA & 62 & 46 & 58 \\
\hline TA & 60 & 46 & 61 \\
\hline TA & 60 & 47 & 59 \\
\hline TA & 60 & 44 & 61 \\
\hline TA & 60 & 44 & 58 \\
\hline Gly & 38 & 64 & 98 \\
\hline Gly & 38 & 60 & 101 \\
\hline Gly & 36 & 63 & 101 \\
\hline Gly & 38 & 61 & 101 \\
\hline Gly & 36 & 62 & 101 \\
\hline AA & 44 & 70 & 98 \\
\hline AA & 43 & 69 & 99 \\
\hline AA & 42 & 68 & 99 \\
\hline AA & 40 & 69 & 98 \\
\hline AA & 42 & 68 & 98 \\
\hline ТP & 30 & 22 & 102 \\
\hline $\mathrm{TP}$ & 30 & 20 & 103 \\
\hline $\mathrm{TP}$ & 26 & 20 & 103 \\
\hline $\mathrm{TP}$ & 26 & 18 & 102 \\
\hline $\mathrm{TP}$ & 26 & 20 & 102 \\
\hline CA & 56 & 42 & 88 \\
\hline $\mathrm{CA}$ & 54 & 39 & 89 \\
\hline CA & 53 & 43 & 89 \\
\hline CA & 54 & 42 & 88 \\
\hline CA & 54 & 42 & 88 \\
\hline
\end{tabular}

